\title{
ITINERÁRIO TERAPÊUTICO DE FAMÍLIAS DE CRIANÇAS COM DEFICIÊNCIA À LUZ DO MODELO TEÓRICO DOS SISTEMAS DE CUIDADOS À SAÚDE
}

\author{
Andreia Chaves Farias ${ }^{1}$, Maria Rosilene Cândido Moreira ${ }^{1}$, Milena Silva Costa ${ }^{1}$, Joseph Dimas \\ de Oliveira1, Antônio Germane Alves Pinto' ${ }^{1}$ e Evanira Rodrigues Maia ${ }^{1}$ \\ 1Universidade Regional do Cariri, Crato, Ceará, Brasil. andreiacf32@gmail.com; rosilene.moreira@ufca.edu.br; \\ milena.costa@ufca.edu.br; josephdimas@hotmail.com; germanepinto@gmail.com; evanira.maia@urca.br
}

\begin{abstract}
Resumo. Introdução: A trajetória percorrida pelos familiares na busca de serviços de atenção à criança com deficiência pode influenciar no cuidado integral dela. Objetivo: Conhecer a atuação do subsistema profissional no cuidado com a saúde de crianças com deficiência. Métodos: Pesquisa qualitativa, desenvolvida em serviços de reabilitação situados no Ceará, Brasil. Participaram 41 familiares de crianças com deficiência, por meio de história oral tópica. Os resultados foram processados pela técnica de análise de similitude proveniente do software IRaMuTeQ e analisados pelo subsistema profissional do modelo teórico dos Sistemas de Cuidados à Saúde. A pesquisa cumpriu os preceitos éticos recomendados. Resultados: Acompanhando o Itinerário Terapêutico, os familiares entraram no subsistema profissional para buscar cuidado na atenção especializada, em um caminho marcado por dificuldades relativas à oferta de serviços de saúde e de educação, $e$ pela ausência de linha de cuidado específica. Desvelou-se a participação de instituições de ensino profissional na oferta de atividades de reabilitação e consulta especializada. Conclusões: $O$ subsistema profissional do Itinerário Terapêutico implicou em prejuízos para o acesso, a equidade, a integralidade e a efetividade do cuidado com crianças com deficiência.
\end{abstract}

Palavras-chave: Crianças com Deficiência; Cuidado da Criança; Atenção à Saúde.

\section{THERAPEUTIC ITINERARY OF FAMILIES OF DISABLED CHILDREN IN THE LIGHT OF HEALTH CARE SYSTEM THEORY}

Abstract. Introduction: The trajectory of family members when looking for care services for disabled children may influence their integral care. Goals: To know the performance of the professional subsystem in the health care for disabled children. Methods: Qualitative research, developed in rehabilitation services located in Ceará, Brazil. In this study, 41 family members of children with disabilities participated, through an interview. The results were processed using the similitude analysis technique from the IRaMuTeQ software and analysed by the professional subsystem of Health Care System model by Arthur Kleinman. The research was in accord with the recommended ethical precepts. Results: Following the Therapeutic Itinerary, family members accessed the professional subsystem in order to have care in specialized care, in a path marked by difficulties related to the provision of health and education services, and to the absence of a specific care line. The participation of professional education institutions in offering rehabilitation and specialized consultation activities was revealed. Conclusions: The professional subsystem of the Therapeutic Itinerary resulted in losses to access, equity, comprehensiveness and effectiveness of care for children with disabilities.

Keywords: Disabled Children; Child Care; Health Care (Public Health). 


\section{INTRODUÇÃO}

As famílias de crianças com deficiência podem apresentar-se isoladas e enfrentar discriminação, o que pode resultar em limitação na utilização dos recursos disponíveis e da inclusão social (Missel, Costa, \& Sanfelice, 2017). Tais problemas ocorrem pelo fato de a criança com deficiência encontrar-se em situação de maior fragilidade e vulnerabilidade, considerando o caráter crônico de sua condição de saúde, a complexidade e a maior demanda por cuidados e serviços, quando comparadas àquelas que vivem sem deficiência (Figueiredo, Sousa, \& Gomes, 2016).

As dificuldades iniciam-se desde o recebimento do diagnóstico da criança pela família, quando são gerados sentimentos como sofrimento, negação, medo e angústia, o que, por si só, já impede ou limita a busca por orientações e atendimentos nos serviços de saúde. Segue-se a ressignificação da identidade, da estrutura e da organização, na qual os papéis e o funcionamento do sistema familiar buscam se adaptar às necessidades da criança (Santos, Marques, \& Souza, 2017).

Os profissionais envolvidos no cuidado devem fortalecer as famílias para o enfrentamento de suas próprias demandas de saúde e as da criança com deficiência. qualidade do cuidado ofertado inicialmente pode influenciar na trajetória percorrida e na busca por atenção. Nesse percurso, as famílias das crianças com deficiência acessam pessoas, lugares, serviços e instituições, para apoio e atendimento de suas demandas, desenhando o que se concebeu chamar de Itinerário Terapêutico (Cerqueira, Alves, \& Aguiar, 2016).

O conceito de Itinerário Terapêutico perpassa por momentos históricos distintos, que vão desde a compreensão de "comportamento do enfermo" (illness behavior), por Mechanic \& Volkart (Siriri, Fava, \& Sonino, 2013) em 1960, em que as escolhas por tratamento se davam sob a lógica de consumo, com ênfase no modelo biomédico e nas demandas por serviços, e ganha importante contribuição na década de 1970 de Arthur Kleinman, médico psiquiatra e antropólogo, que deu ênfase à influência cultural nas escolhas do doente relativas ao percurso de cuidado (Kleinman, 1978; Siqueira et al., 2016).

O modelo teórico dos Sistemas de Cuidados à Saúde de Kleinman apresenta dois conceitos importante: o de modelos explicativos, que refere-se às concepções sobre a doença e as formas de tratamento utilizadas por todos aqueles engajados em um processo clínico, e o de sistema de atenção à saúde, que refere-se ao locais onde os indivíduos buscam soluções 
para seus problemas de saúde e são classificados em três setores (profissional, constituído pelas práticas formais de exercício da medicina, como biomedicina e homeopatia; o popular, que são os cuidados caseiros e o autocuidado; ou o folk, que corresponde a práticas místicas e religiosas de cuidados). O Itinerário Terapêutico, nesse caso, é o percurso que os indivíduos vivenciam nos sistema de atenção à saúde, tomando por base seu modelo explicativo do processo saúde-doença (Cabral, Martinez-Hemáez, Andrade, \& Cherchiglia, 2011).

Conhecer o Itinerário Terapêutico das famílias de crianças com deficiência possibilita identificar os componentes acessados, os desafios e potencialidades dos serviços da rede na oferta de cuidados e os aspectos que influenciam nas escolhas do cuidado na Rede de Atenção à Saúde (RAS), por meio do planejamento da atenção integral à saúde da criança com deficiência (Brasil, 2014).

A Rede de Cuidados à Pessoa com Deficiência deve ampliar acesso a serviços, qualificar o atendimento e vincular pessoas com deficiência e famílias aos pontos de atenção de modo articulado, com acolhimento e classificação de risco. Priorizam-se acompanhamento do recém-nascido de alto risco até 2 anos de vida, tratamento das crianças com deficiência e suporte adequado às famílias nas linhas de cuidado, definidas como fluxos assistenciais seguros e que garantam atendimento de saúde por meio de ações integrais (Brasil, 2012; Franco, Santos, \& Salgado, 2011).

A dissonância é que há fragilidades nas linhas de cuidado com a criança com deficiência. Evidencia-se dificuldade de acesso e acompanhamento em serviços de reabilitação não regulados pelo Sistema Único de Saúde (SUS), sem coordenação do cuidado e/ou vinculação com a Atenção Primária em Saúde (APS), falhas no acolhimento, desarticulações nos serviços que constituem fatores agravantes e impeditivos para 0 cuidado efetivo e integral.

A pesquisa em tela objetiva conhecer a atuação do subsistema profissional no cuidado com a saúde das crianças com deficiência.

\section{METODOLOGIA}

Trata-se de pesquisa qualitativa, por possibilitar a identificação do universo de significados, crenças, valores, atitudes e aspirações dos entrevistados, além da constituição de um 
espaço mais profundo das relações, dos processos e dos fenômenos. Esse modelo de investigação exerce primazia sobre o produto final e permite a incorporação de aspectos do significado e da intencionalidade como próprio das ações e das estruturas sociais, sendo estas tidas como construções humanas significativas (Minayo, 2014).

Este tipo de pesquisa se adequa à proposta em tela, uma vez que se pretendeu investigar aspectos subjetivos do subsistema profissional do Itinerário Terapêutico das famílias de crianças com deficiência, que só poderiam ser desvelados por meio de relatos e vivências dos envolvidos.

A pesquisa foi desenvolvida em um Núcleo de Estimulação Precoce (NEP) e na Associação de Pais e Amigos dos Excepcionais (APAE), situados em municípios do interior cesarense brasileiro. Ambos os locais são serviços especializados de reabilitação e de inserção social.

O acesso da criança e da família ao NEP acontece por encaminhamento das Unidades Básicas de Saúde ou demais pontos de atenção, via central de marcação. É realizada consulta com neuropediatra, que avaliada e diagnostica a criança, iniciando a elaboração e a implantação do plano terapêutico interdisciplinar. O NEP atende crianças de zero a 3 anos de idade, com distúrbios do desenvolvimento neuropsicomotor (ADNPM), seja por microcefalia ou por outras patologias, como paralisia cerebral, síndrome de Down, prematuridade, dentre outras (Ceará, 2017).

A APAE é uma organização social com o propósito de promover atenção integral à pessoa com deficiência intelectual e múltipla. Acompanha pessoas com deficiência em todo ciclo de vida e nas diversas especialidades, da prevenção à reabilitação. Conta com equipe multidisciplinar, composta por terapeuta ocupacional, psicóloga, fonoaudióloga, fisioterapeuta, enfermeira e assistente social, para atendimento a crianças de zero a 11 anos 11 meses e 29 dias (Apae, 2019).

Os participantes da pesquisa foram os familiares das crianças com deficiência na faixa etária de zero a 9 anos, 11 meses e 29 dias, inseridos nos serviços de reabilitação lócus da pesquisa, nos meses de maio a novembro de 2019.

À época da coleta de dados, estavam cadastradas nesses serviços 134 crianças com deficiência. Adotaram-se os seguintes critérios de inclusão: estar envolvido no cuidado diário da criança com deficiência e residir em município localizado no sul do Ceará. Os critérios de 
exclusão foram: familiares que apresentassem deficiência verbal e/ou intelectual que impossibilitasse o relato e participação na pesquisa.

Nesse estudo, adotou-se a saturação do tipo code saturation, que é definida como o ponto quando problemas adicionais não são identificados, e o sistema de codificação dos dados é estabilizado, ou seja, não surgem temas novos nas falas dos entrevistados (fontes primárias). As entrevistas foram encerradas após os códigos/temas das falas mostrarem-se repetidos e sem nenhum dado adicional novo (Hennink, Kaiser, \& Marconi, 2017).

Utilizaram-se dois instrumentos para coleta de dados, que foram um roteiro de entrevista estruturada, contendo informações sociodemográficas e de saúde do familiar e da criança, e a entrevista em profundidade, com foco nas experiências vividas no Itinerário Terapêutico da criança com deficiência, com a pergunta norteadora: "Peço-lhe que me fale sobre a trajetória percorrida na busca por cuidados para a criança com deficiência”. A pergunta foi acrescida de questionamentos, de modo a atender aos objetivos do estudo.

A entrevista em profundidade foi fundamentada no método da história oral, por meio da técnica de trajetória de vida, entendida como parte de uma história de vida, em que o relato é focalizado em um momento ou experiência de vida, a qual vem ao encontro do objeto de pesquisa (Minayo, 2014). Esse método permitiu elucidar experiências vividas pelas famílias de crianças com deficiência no Itinerário Terapêutico em uma perspectiva de compreensão da dinâmica das relações.

Os relatos de história de vida tópica foram ouvidos e transcritos para um arquivo no programa LibreOffice Writer, versão 5.4 , sendo codificados conforme a sequência numérica das entrevistas e o respectivo serviço especializado no qual cada familiar encontrava-se vinculado, constituindo o banco de dados do estudo (corpus textual), que foi submetido a uma análise de similitude, com o auxílio do programa informático IRaMuTeQ, versão 0.7 alpha 2. Este software, gratuito, com código aberto, ancora-se no software $\mathrm{R}$ e na linguagem de programação Python, para viabilizar diferentes modos de análise, desde os mais simples, como a lexicografia básica, até análises multivariadas (Camargo \& Justo, 2013), como a adotada neste estudo.

A análise de similitude, que toma por base a teoria dos grafos, possibilita identificar as coocorrências entre palavras, as quais indicam a conexidade entre elas, auxiliando na 
identificação da estrutura do conteúdo do corpus textual. Este tipo de análise permite ainda identificar partes comuns e especificidades do material, em função das variáveis descritivas identificadas na análise (Ratinaud \& Marchand, 2012; Camargo \& Justo, 2013). Delineada sob o formato de árvore de coocorrências, apresenta-se por uma imagem contendo nuvens coloridas interligadas por vértices, representando os grupos de palavras mais associadas entre si (Camargo \& Justo, 2018), que variam em tamanho e posição, e anunciam diferentes graus de interconexão de subtemas - no caso presente, os aspectos do itinerário terapêutico das famílias investigadas.

O modelo teórico dos Sistemas de Cuidados à Saúde subsidiou a análise interpretativa da árvore de similitude e constituiu o arcabouço teórico-metodológico que balizou o estudo. Este referencial é constituído por três subsistemas sociais, que consistem em subsistema popular, folclórico (folk) e profissional. Para este estudo, contemplou-se o subsistema profissional, do qual fazem parte os profissionais da medicina científica ocidental e de sistemas de cura tradicionais (Kleinman, 1978). Tal escolha deve-se ao fato dessa análise favorecer a identificação das ações em saúde das crianças com deficiência nos serviços, por meio do itinerário terapêutico.

A pesquisa obedeceu ao disposto nas resoluções 466/2012 e 510/2016 do Conselho Nacional de Saúde (Brasil, 2013), estando aprovada pelo Comitê de Ética e Pesquisa da Universidade Regional do Cariri (URCA), sob o parecer 3.325.364.

\section{RESULTADOS E DISCUSSÃO}

Participaram da pesquisa 41 familiares mulheres, sendo 37 mães-genitoras, duas mãesadotivas, uma tia e uma avó. A maioria era do lar, com idade entre 30 e 39 anos, Ensino Médio completo, católica, casada/em união estável e renda familiar de um salário mínimo do Brasil em 2019 (R\$ 998,00), proveniente do Benefício de Prestação Continuada (BPC), por vezes complementada por trabalho informal e esporádico. Destas, 20 foram entrevistadas no NEP e 21 na APAE.

Embora a deficiência não se resuma a uma perspectiva biomédica, a definição de sua etiologia contribui para melhor atenção às necessidades biológicas da criança que a apresenta e do percurso terapêutico a ser seguindo, possibilitando um plano de atenção efetivo no subsistema profissional de cuidados. Ademais, fornece à família informações 
importantes, que ajudam na aceitação familiar (Melo, Pilotto, Rodrigues, Silva, \& Germano, 2018).

O perfil apresentado coaduna-se com a reconstrução dos Itinerários Terapêuticos das famílias no subsistema profissional no cuidado com a saúde das crianças com deficiência. Tais Itinerários Terapêuticos são marcados por inexistência ou falhas nos fluxos assistenciais, acessibilidade, articulação, ordenação e integralidade da rede de cuidados. As falas são representativas da situação evidenciada e descrita na história oral das cuidadoras. Na Figura 1, a árvore de similitude do corpus do Itinerário Terapêutico traz uma representação gráfica do subsistema profissional percorrido por familiares de crianças com deficiência.

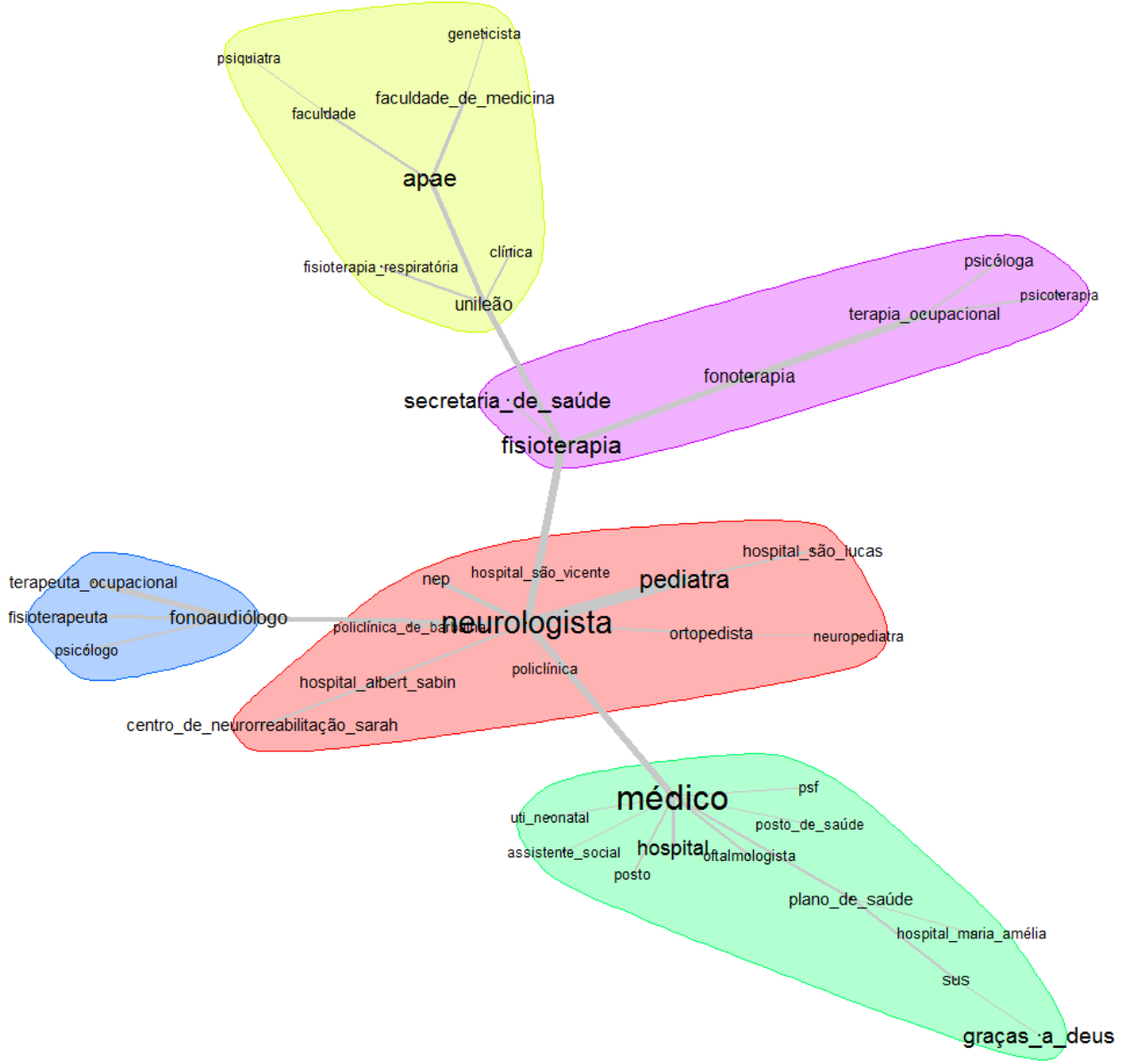

Figura 1. Árvore de similitude do Itinerário Terapêutico de familiares de crianças com deficiência, 2019. 
Há evidências de profissionais e serviços que se apresentam como porta de entrada no sistema de saúde para essas crianças, composta pelo neurologista, especialista que assume uma posição central de investigação diagnóstica e acompanhamento da criança no Itinerário Terapêutico; pelo pediatra, que aparece em uma relação de grande proximidade com o neurologista, evidenciando uma ação clínica conjunta entre os dois especialistas, em um contínuo de cuidado.

Eu só tinha tido uma consulta com o neurologista, porque lá a demanda era muito grande; aí eu passei para a pediatra, que a microcefalia dela estava confirmada... (E05, NEP)

Neste plano, os termos "médico", do qual flui "secretaria de saúde"- a qual aparece em uma perspectiva de sistema de acesso regulado, sistema logístico -, "posto de saúde", "neuropediatra" e "oftalmologista" evidenciam os fluxos primários de cuidado, ao mesmo tempo em que estabelece uma relação de proximidade com o "neurologista". Os termos "hospital pediátrico municipal" e "NEP" representam acesso a instituições de cuidado secundário. São colocados em evidência os termos "plano de saúde" e "SUS", deixando claro que os familiares caminham por serviços do SUS e de saúde suplementar na busca por atenção, o que pode estar relacionado à fragilidade da linha de cuidado.

Foi como nós fizemos, por intermédio do oftalmologista que a gente encontrou o outro oftalmologista, fizemos a consulta com ele, e ele falou da cirurgia. Ele mesmo que faz a cirurgia no hospital de referência. (E18, NEP)

As consultas foram particulares; nunca procurei o SUS; como é o posto de saúde tem uma área muito grande lá [no bairro], ficaria mais difícil para mim e como ele já tinha o plano de saúde, eu achei muito mais prático usar o plano. (E40, APAE)

Da comunidade central da árvore de similitude fluem três comunidades. A primeira traz em destaque os termos "APAE" em aproximação direta com "faculdade de medicina" - por meio da qual estabelece comunicação com a "geneticista" -, "clínica escola" e "terapia ocupacional" - e, de forma secundária, com "clínica de fisioterapia", evidenciando as instituições envolvidas e atividades terapêuticas a que são submetidas às crianças. A segunda comunidade diz respeito aos principais profissionais de saúde não médicos envolvidos nas atividades de reabilitação: o "fonoaudiólogo" e o "terapeuta ocupacional". A terceira comunidade apresenta instituições da atenção secundária e terciária de referência, representada pelos termos "hospital pediátrico estadual" e "centro de neurorreabilitação". 
A mãe fica mesmo angustiada, porque eu saí com a carta daqui da Apae para ir para a faculdade, e quando cheguei lá, disseram que não tinha mais agenda para a neurologista atender, porque ela atende convênio e atende algumas crianças por mês, e só tinha ela. (E37, $A P A E)$

Depois que ele entrou no NEP foi que teve a avaliação com os profissionais, mas o neurologista não, só a fonoaudióloga, terapeuta ocupacional, a enfermeira e a fisioterapeuta. (E04, NEP)

Lá [NEP] foi diagnosticado, e desde então o acompanhamento é lá, além do centro de neurorreabilitação. (E04, NEP)

O itinerário pesquisado destacou aspectos como presença marcante das clínicas escolas na reabilitação e consultas especializadas, por meio de encaminhamentos informais, sem a devida articulação com os demais pontos de atenção; deslocamento da APS, a qual se apresentou em posição secundária, com implicação para a coordenação do cuidado e para o estabelecimento de fluxos seguros e efetivos e o défice ou inexistência na atenção ofertada em alguns serviços, ocasionando ausência de fluxos de cuidado que orientassem os familiares para um percurso seguro e efetivo.

Destaca-se que o desenho do Itinerário Terapêutico desvelado pelos familiares das crianças com deficiência desse estudo foi caracterizado por serviços de saúde públicos, privados (clínicas escolas) e filantrópicos, com presença de profissionais de saúde, médicos e não médicos. Revelou que a entrada no subsistema profissional aconteceu por meio da atenção secundária, com o envolvimento dos profissionais médicos especialistas (neurologistas, pediatras e neuropediatras) já que, por meio deles, foram realizadas as devidas investigações diagnósticas e/ou encaminhamentos da criança aos serviços de reabilitação (neuropediátrica).

O percurso percorrido identificou que a busca por assistência à criança com deficiência é um processo difícil e se caracteriza por uma peregrinação solitária de familiares por serviços e profissionais de saúde, por vezes, evidenciando inexistência ou fragilidade de uma linha de cuidado efetiva, além do descontínuo do cuidado iniciado na maternidade.

Observou-se, por meio da reconstrução oral dos itinerários percorridos, que os familiares descrevem e atribuem significado às experiências vivenciadas na realidade social, na busca do cuidado com a saúde prevista no SUS, por meio das RAS. No entanto, a árvore de similitude detalhou dificuldades na possibilidade de acesso e da continuidade da assistência na rede de cuidados. A história contada pelos familiares demonstra percurso marcado por 
significados conflituosos atribuídos aos arranjos governamentais e não governamentais instituídos, no qual a APS não exerce seu papel ordenador e coordenador do cuidado, por meio da comunicação com os demais pontos de atenção.

A vivência dos familiares destaca que a continuidade e a integralidade do cuidado com a criança egressa da maternidade/unidade neonatal são oportunizadas pela comunicação entre esta rede e a APS, porém, a fragilidade nessa comunicação aponta para a transferência de responsabilidade da contrarreferência para o cuidador, comprometendo a continuidade da atenção. Essa falha de comunicação posterga o diagnóstico adequado e leva mães e familiares a serem os primeiros a perceberem os sinais de atraso no desenvolvimento da criança. Assim, a busca inicia-se por um diagnóstico. Esta busca acontece motivada pelo desejo de resolução das necessidades da criança e cura. No entanto, diante do diagnóstico fechado, os familiares se deparam com o caráter permanente da deficiência, surgindo novos questionamentos e caracterizando um momento de crise familiar (Franco, 2016).

O sofrimento dos pais, relatado nos discursos dos cuidadores, diante do diagnóstico de deficiência em um filho, é real e perpassa por uma vivência de luto (a morte do filho idealizado, sem doença). Esse processo é permeado por um misto de sentimentos, como choque, medo, angústia, tristeza, raiva e culpa, que se apresentam em perspectiva de enfrentamento da realidade (Oliveira \& Poletto, 2015).

É uma busca [pelo diagnóstico] que você faz sem querer ver o fim, porque você tem medo da resposta, por mais que você tenha a certeza que vai ser, porque tudo dizia e eu não queria aceitar. (E30, NEP)

E não adianta a pessoa se desesperar; é confiar e entregar a Deus, que tudo vai dar certo; tudo, tudo vai dar certo quando a gente confia em Deus; e graças a Deus, até hoje deu certo. Até hoje estou aqui com ela cuidando dela, e cuidarei dela até o dia que Deus quiser, que Deus permitir. (E39, APAE)

Em sua teoria, Kleinman (1978) elucida que, apesar de a cura constituir-se em uma das principais funções do sistema de saúde, nem sempre ela é possível. Assim, deve-se "ajustar as expectativas, crenças, comportamento e avaliações do resultado", principalmente do ponto de vista da eficácia terapêutica de determinadas doenças, como a deficiência. Desse modo, a competência e a sensibilidade do profissional de saúde responsável pela comunicação do diagnóstico são imprescindíveis para o adequado apoio familiar e influenciam na elaboração dos sentimentos vivenciados pelos pais e nos papéis a serem 
desempenhados por eles, na direção do cuidado e da inclusão (Oliveira \& Poletto, 2015). Dada a complexidade e as características do cuidado com a criança com deficiência, o subsistema profissional apresenta-se primordial no Intinerário Terapêutico para o desenvolvimento das práticas de cuidados.

No entanto, ao fazer um paralelo entre o itinerário desenhado e a proposição de Rede de Cuidados à Pessoa com Deficiência, foi possível inferir fragilidade de uma linha de cuidado locorregional para a atenção integral das crianças com deficiência evidenciada no estudo, o que contribuiu para a construção de fluxos de cuidado adaptados por profissionais, na perspectiva de prover o cuidado, assemelhando-se ao que Mendes (2016) chamou de "perambulação desorientada" dos familiares das crianças com deficiência por serviços de saúde.

É importante destacar que, para além do cuidado de saúde, e ainda no itinerário de cuidado, os familiares encontraram lacunas nas instituições do subsistema profissional. A configuração da arena profissional é fruto do modelo construído na realidade social, no qual aspectos sociais, econômicos, psicológicos e culturais exercem fundamental pressão na formulação, na implementação e na materialização dos cuidados ofertados.

Dentre as funções exercidas pelos sistemas de cuidado de saúde, destacando o subsistema profissional, Kleinman (1978) destaca que a comunicação é fator determinante para o desfecho satisfatório dos problemas de saúde e influi sobremaneira na adesão, na adequação e na satisfação dos usuários com os serviços de saúde. No cenário estudado, as falhas de comunicação da rede de cuidados influenciaram negativamente nos resultados clínicos e na atenção prestada a crianças com deficiência.

\section{CONCLUSÕES}

O papel do subsistema profissional é predominante no Itinerário Terapêutico percorrido pelas famílias para acesso ao cuidado de saúde de crianças com deficiência. Porém, esse subsistema é caracterizado como deficitário, por não promover a integração entre os serviços da rede. Os familiares não visualizaram um fluxo assistencial estabelecido para acessar profissionais responsáveis pela assistência a crianças com deficiência, implicando em fragilidade estrutural e na organização da Rede de Atenção à Saúde quanto a acesso, equidade, integralidade e efetividade do cuidado. 
No desenvolvimento de escopos investigativos para cotidianos assistenciais, o olhar qualitativo pode se limitar pela interpretação focal de um contexto específico, mas se amplia pela profundidade intencional das relações. Neste estudo, inova-se com a instrumentalização eletrônica e sistemática das informações que, aliadas às vivências entre participantes e pesquisadores, exaltam as necessidades de saúde e cuidado como alvo da atenção à saúde.

Agradecimentos. ao Ministério da Saúde do Brasil pelo apoio ao Programa de Pós-Graduação da Rede Nordeste de Formação em Saúde da Família (RENASF).

\section{REFERÊNCIAS}

Associação de Pais e Amigos dos Excepcionais (Apae). (2019). Quem Somos. Federação Nacional das Apaes. Brasília. Disponível em: http://apaebrasil.org.br/

Brasil. Ministério da Saúde. (2014). Implantação das Redes de Atenção à Saúde e outras estratégias da SAS. Brasília, DF: Ministério da Saúde. Disponível em: http://bvsms.saude.gov.br/bvs/publicacoes/implantacao_redes_atencao_saude_sas.pdf

Brasil. Ministério da Saúde. (2013). Resolução no 466, de 12 de dezembro de 2012. Brasília, DF: Diário Oficial da União. Disponível em: https://conselho.saude.gov.br/resolucoes/2012/Reso466.pdf

Brasil. Ministério da Saúde. (2012). Portaria n.793, de 24 de abril de 2012. Institui a Rede de Cuidados à Pessoa com Deficiência no âmbito do Sistema Único de Saúde. Brasília, DF: Diário Oficial da União. Disponível em: http://bvsms.saude.gov.br/bvs/saudelegis/gm/2012/prt0793_24_04_2012.html

Cabral, A. L. L. V., Martinez-Hemáez, A., Andrade, E. I. G, \& Cherchiglia, M. L. (2011). Itinerários terapêuticos: o estado da arte da produção científica no Brasil. Ciência \& Saúde Coletiva, 16(11), 4433-4442.

Camargo, B. V. \& Justo, A. M. (2013). IRAMUTEQ: um software gratuito para análise de dados textuais. Temas em Psicologia, 21(2), 513-518.

Ceará, P. M. I. C. (2017). A experiência do Estado do Ceará no enfrentamento à síndrome congênita do Zika Vírus. Campinas: Pontes Editores. Disponível em: https://www.ceara.gov.br/wpcontent/uploads/2019/03/WEB-LIVRO-1-Programa-mais-infância-ceará.pdf

Cerqueira, M. M. F., Alves, R. O., \& Aguiar, M. G. G. (2016). Experiências vividas por mães de crianças com deficiência intelectual nos itinerários terapêuticos. Ciência \& Saúde Coletiva, 21(10), 3223-3232.

Figueiredo, S. V., Sousa, A. C. C., \& Gomes, I. L. V. (2016). Menores com necessidades especiais de saúde e familiares: implicações para a enfermagem. Revista Brasileira de Enfermagem, 69(1), 88-95. 
Franco, C. M., Santos, A. S., \& Salgado, M. F. Manual do gerente: desafios da média gerência na saúde. In: Pessoa, L. R., Santos, E. H. A., \& Torres K. B. R. O., orgs. (2011). Manual do gerente: desafios da média gerência na saúde. Rio de Janeiro: Escola Nacional de Saúde Pública Sérgio Arouca.

Franco, V. (2016). Tornar-se pai/mãe de uma criança com transtornos graves do desenvolvimento. Educar em Revista, 59(1), 35-48.

Hennink, M. M., Kaiser, B. N., Marconi, V. C. (2017). Code saturation versus meaning saturation: how many interviews are enough? Qualitative Health Research, 27(4), 591-608.

Kleinman, A. (1978). Concepts and a model for the comparison of medical systems as cultural systems. Social Science \& Medicine, 12(2B), 85-95.

Siriri, L., Fava, G. A., Sonino, N. (2012). The unifying concept of illness behavior. Psychotherapy and Psychosomatics, 82, 74-81.

Melo, D. G., Pilotto, R. F., Rodrigues, S. A., Avó, L. R. S., \& Germano, C. M. R. (2018). Investigação etiológica nas situações de deficiência intelectual ou atraso global do desenvolvimento. Revista Saúde e Desenvolvimento Humano, 6(3), 73-85.

Mendes, E. V. (2016). O acesso à atenção primária à saúde. Brasília, DF: Conselho Nacional dos Secretários de Saúde.

Minayo, M. C. S. (2014). O desafio do conhecimento. São Paulo: Hucitec.

Missel, A., Costa, C. C., \& Sanfelice, G. R. (2017). Humanização da saúde e inclusão social no atendimento de pessoas com deficiência física. Trabalho, Educação e Saúde, 15(2), 575-597.

Oliveira, I. G., \& Poletto, M. (2015). Vivências emocionais de mães e pais de filhos com deficiência. Revista da SPAGESP, 16(2), 102-119.

Ratinaud, P., \& Marchand, P. (2012). Application de la méthode ALCESTE à de "gros" corpus et stabilité des "mondes lexicaux": analyse du "Cable-Gate" avec IraMuTeQ. In: Actes des 11eme Journées internationales d'Analyse statistique des Données Textuelles. pp. 835-844. Liège, Belgique.

Santos, K. H., Marques, D., \& Souza, A. C. (2017). Crianças e adolescentes com paralisia cerebral: análise sobre longitudinalidade do cuidado. Texto \& Contexto - Enfermagem, 26(2), e00530016 .

Siqueira, S. M. C., Jesus, V. S., \& Camargo, C. L.(2016). Itinerário terapêutico em situações de urgência e emergência pediátrica em uma comunidade quilombola. Ciência \& Saúde Coletiva, 21(1),179-189. 